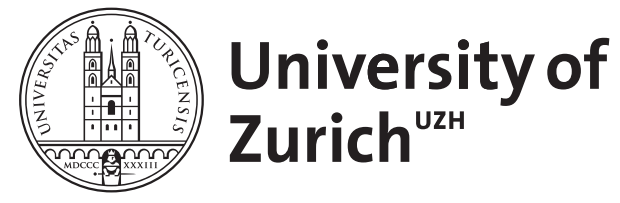

\title{
Reward, value, and salience
}

Kahnt, Thorsten ; Tobler, Philippe N

\begin{abstract}
Value and salience are key variables for associative learning, decision-making, and attention. In this chapter we review definitions of value and salience, and describe human neuroimaging studies that dissociate these variables. Value increases with the magnitude and probability of reward but decreases with the magnitude and probability of punishment, whereas salience increases with the magnitude and probability of both reward and punishment. Moreover, salience may be particularly enhanced in situations with probabilistic as opposed to safe outcomes. At the behavioral level, both value and salience independently accelerate behavior. At the neural level, value signals arise in striatum, orbitofrontal and ventromedial prefrontal cortex, and superior parietal areas, whereas magnitude-based salience signals arise in the anterior cingulate cortex and the inferior parietal cortex. By contrast, probability-based salience signals have been found in the ventromedial prefrontal cortex. In conclusion, the related nature of value and salience stresses the importance of disentangling both variables experimentally.
\end{abstract}

DOI: https://doi.org/10.1016/B978-0-12-805308-9.00009-9

Posted at the Zurich Open Repository and Archive, University of Zurich

ZORA URL: https://doi.org/10.5167/uzh-147578

Book Section

Accepted Version

Originally published at:

Kahnt, Thorsten; Tobler, Philippe N (2017). Reward, value, and salience. In: Dreher, Jean-Claude; Tremblay, Léon. Decision Neuroscience. Amsterdam: Elsevier, 109-120.

DOI: https://doi.org/10.1016/B978-0-12-805308-9.00009-9 


\section{Reward, VALUe AND SALIENCE}

Thorsten Kahnt ${ }^{1} \&$ Philippe N. Tobler ${ }^{2 *}$

${ }^{1}$ Department of Neurology

Northwestern University Feinberg School of Medicine

303 E Chicago Avenue

Chicago IL 60611

USA

Phone: +1 3125032896

Email: thorsten.kahnt@northwestern.edu

${ }^{2}$ Department of Economics

University of Zurich

Blümlisalpstrasse 10

8006 Zürich

Switzerland

Phone: +41 446343745

Email: phil.tobler@econ.uzh.ch

* Senior/corresponding author

\section{KEYWORDS}

Value, magnitude, probability, reward, punishment, salience, attention, associative learning, fMRI 


\begin{abstract}
Value and salience are key variables for associative learning, decision-making, and attention. In this chapter we review definitions of value and salience, and describe human neuroimaging studies that dissociate these variables. Value increases with the magnitude and probability of reward but decreases with the magnitude and probability of punishment, whereas salience increases with the magnitude and probability of both reward and punishment. Moreover, salience may be particularly enhanced in situations with probabilistic as opposed to safe outcomes. At the behavioral level, both value and salience independently accelerate behavior. At the neural level, value signals arise in striatum, orbitofrontal and ventromedial prefrontal cortex, and superior parietal areas, whereas magnitude-based salience signals arise in the anterior cingulate cortex and the inferior parietal cortex. By contrast, probability-based salience signals have been found in ventromedial prefrontal cortex. In conclusion, the related nature of value and salience stresses the importance of disentangling both variables experimentally.
\end{abstract}




\section{INTRODUCTION}

One of the most adaptive functions for humans and animals is the ability to predict future outcomes. This ability allows us to prepare and execute appropriate actions to obtain rewards and avoid punishments. For example, the sight of fruits hanging from a tree allows us to approach the tree and harvest the fruits. Similarly, if we have learned about the likely consequences of this stimulus, the sight of a leopard in a tree allows for avoidance behavior. In other words, the appropriate use of information provided by predictive stimuli allows for survival-promoting, adaptive behavior. Such behavior is also beneficial in evolutionary terms, by providing an advantage over other members of the species who have learned the meaning of the stimuli less well, and who as a consequence get to eat less fruit and are more likely to fall prey to a leopard [1].

A hallmark of adaptive behavior is that it relates stimuli in a meaningful way to their predicted outcomes. In particular, the magnitude of the predicted outcome is an important factor that influences behavior. For example, the sight of many fruits on a tree signals more reward and may increase the probability of approach compared to the sight of fewer fruits. Similarly, an adult leopard may be avoided more resolutely than a baby leopard. Despite the different responses they elicit, both the tree with plenty of fruits and the adult leopard will capture more attention and cognitive resources than the tree with just a few fruits and the baby leopard. Increased attention in turn will reduce the latency of approach and avoidance responses. These orderly relations between stimuli and predicted outcomes can be captured by two theoretical concepts: value and salience. Roughly speaking, value guides appropriate action selection in a magnitude-dependent fashion, whereas salience captures attention according to the overall importance of the stimulus. 
Value arises from the intrinsic meaning of two different types of predicted outcomes: rewards and punishments. Rewards have positive value and are approached, whereas punishments have negative value and are avoided. Thus, value is a signed currency that varies in a monotonic fashion from negative to positive. In contrast, salience is an unsigned currency, and the more negative or the more positive the predicted outcome, the more salient it is and the more attention it will draw. Thus, in the scheme proposed here, salience relates to value such that the absolute value of the predicted outcome determines its salience, irrespective of whether the predicted outcome is a reward or a punishment [2,3]. For example, if the absolute value of a banana is the same as the absolute value of an intimidating display of a conspecific, then the two have equal salience. The fact that the definitions of value and salience are related to each other may explain why the literature has not always separated them in empirical studies $[4,5]$.

The predictive relations between stimuli and outcomes have to be learned from experience. It should therefore come as no surprise that learning theory has much to say about predicted value and salience [6-8]. In particular, associative learning theory describes the mechanisms with which predictive stimuli become valuable [9] and salient [10-12]. Moreover, learning theory has provided several, not mutually exclusive, definitions of salience and suggested ways in which they could be combined [13].

This chapter reviews how value and salience are defined by learning theory, how they can be distinguished from each other experimentally, and how they are processed in the human brain to mediate adaptive behavior. Importantly, we focus on predictive (learned) value and salience rather than on experienced (outcome-related) value and salience [e.g., 14]. Moreover, this chapter only discusses human studies, and the reader is referred to different chapters of 
this book covering the neural processing of value and salience in animals (see chapters 2-5) [see also 15].

\section{VALUE}

\subsection{DEFINITION AND BEHAVIORAL FUNCTION}

Value has been defined in many different ways and is an important concept for various scientific disciplines such as psychology, economics, and ecology. The importance of the concept arises from its various functions. Value guides choice behavior [16]. In particular, stimuli with positive value elicit approach, whereas stimuli with negative value elicit avoidance behavior. In addition to guiding decisions, value constituting rewards and punishments also elicit associative learning [9] and are associated with emotions, including pleasure and pain [17]. Here we consider learned value, that is, the value that a previously neutral stimulus has acquired through association with a reward or punishment. The computational and neural processes by which predictive stimuli acquire value through associative learning are discussed in different chapters of this book.

At the most general level, value can be defined as the capacity of a stimulus or goal to elicit effort exertion. We exert energy (i.e. work) to obtain stimuli with positive value and to avoid stimuli with negative value. In addition, we work harder with more highly valued stimuli than with less highly valued stimuli. Experimenters can harness the monotonic relationship between behavior and predicted outcomes, and infer the value of the outcome by the strength, latency, or probability of the response elicited by the outcome predictive stimulus (i.e., the conditioned response). For example, stimuli predictive of larger or more likely rewards elicit stronger approach behavior in rats (e.g., as measured by their running speed down an alley) 
than stimuli predictive of smaller or less likely rewards [18]. Accordingly, experimenters can take the running speed elicited by the different predictive stimuli as a proxy for value.

Work or energy exertion in the above definition is meant in a broad sense and not restricted to specific types of learned actions. Still, it applies most naturally to instrumental conditioning, i.e. situations in which delivery of the outcome depends on whether a specific response is performed [8]. Nevertheless, also with Pavlovian conditioning, i.e. in situations in which the delivery of the outcome is independent of any response production, conditioned responses occur and scale with value. For example, dogs salivate more to stimuli that predict more meat powder [7], and thus, Pavlovian responses can also be taken as a proxy for value.

In the definition above, value is a subjective variable that has to be inferred from how individuals respond to stimuli. However, value directly relates to objective parameters that can be controlled experimentally. For instance, value increases with increasing reward magnitude and decreases with increasing punishment magnitude (Fig. 1A). Thus, a stimulus predicting two cherries is more valuable than a stimulus predicting one cherry. Similarly, value increases with increasing probability of reward and decreases with increasing probability of punishment (Fig. 1B). Accordingly, a stimulus predicting a cherry with $100 \%$ is more valuable than a stimulus predicting a cherry with $50 \%$, which in turn is more valuable than a stimulus predicting a cherry with $0 \%$. For simplicity, Figure 1 depicts value as a linear function of magnitude and probability but empirically, this relation is often found to be nonlinear, at least for large magnitudes and probabilities close to 0 and 1 [16]. 
The value functions depicted in Figure 1 span the whole range of reward and punishments. This implies that a true value signal not only distinguishes rewards and punishments according to their positive versus negative valence but that a difference of two value units in the punishment domain is represented similarly to a difference of two units in the reward domain. Such value coding on a common scale is a requirement for making computations across the entire range of possible values and allows us to determine the integrated value of stimuli predicting mixed outcomes (i.e., losses and gains, rewards and punishments, or costs and benefits).

The definition of value as a function of magnitude and probability has been taken up in various economic theories suggesting different ways in which probability and magnitude should be, or are, combined during choice (expected value, expected utility, prospect; [for review 19]). For instance, by multiplying the magnitude of an outcome by the probability with which it occurs, the two variables are integrated to form the expected value. In neoclassical economic theory [e.g., 20], value (or utility) has become a placeholder that can capture overt choice behavior (revealed preferences) if that behavior obeys a set of basic principles (axioms, such as transitivity: if one prefers A over B and B over C then one ought to prefer A over C). In other words, as long as choice behavior follows these principles, it can be described as if decision makers maximize a hypothetical value function. Although research in behavioral economics, psychology, and neuroscience has repeatedly found empirical deviations from this normative neoclassical ideal [e.g., 16], these deviations do not contradict the basic notion that value is a function of magnitude and probability. 
Reinforcement learning theory also recognizes that the value of the predicted outcome increases with magnitude and probability of occurrence. Accordingly, the strength of the predictive response increases with magnitude and probability. Value learning corresponds to the gradual adjustment of predictions until the predicted value reflects the experienced value [for review 21]. At the end of each trial [9], or at each moment in time [22], the organism is thought to compute a prediction error that corresponds to the value difference between the actual outcome (or state) and the predicted outcome (or state). The prediction error is used to update the previous prediction in order to generate a more accurate prediction. The ultimate goal is to adjust the predictions such that they are proportional to the value of the outcome, both for magnitude and probability. Thus, at the end of learning, value predictions correspond to the value functions depicted in Figure 1.

So far we have considered one single type of value learning for a single use, namely stimulusoutcome learning for the prediction of rewards and punishments. However, it is likely that independent valuation systems can be employed in parallel that are differentially suited to handle variable versus stable environments. For example, a goal-directed learning system may be based on a mental model of the world, and represent the long-term value of outcomes resulting from a number of different future actions and states. Alternatively, a habitual learning system may represent experience-based value predictions of the immediate state without the need of a model. In other words, different value systems may use different mechanisms to learn different value predictions and to support habitual or goal-directed behavior [23]. 


\subsection{VALUE SIGNALS IN THE HUMAN BRAIN}

Functional neuroimaging has revealed predicted outcome magnitude and probability signals in the human striatum and midbrain, consistent with results from studies using single cell recordings [24]. The striatal signals are reminiscent of the responses emitted by single dopamine neurons which increase firing with both predicted reward magnitude and probability $[25,26]$. In one human neuroimaging study [27], subjects learned the meaning of visual stimuli that were graded in two dimensions: color (between light yellow and dark red) and shape (between one and five circles). One dimension was associated with gradations in reward magnitude, and the other with gradations in probability (across subjects, the dimension to probability or magnitude assignment and the increasing/decreasing direction of the dimensions was counterbalanced). FMRI data were acquired after learning was completed. In each trial, one stimulus was shown and subjects indicated with a button press where on the screen it was displayed. Stimulus-induced activations in the striatum increased with probability, magnitude, and their combination. Similar magnitude- and probability-related striatal activations have been reported for a variety of tasks [28-44]. For instance with magnitude, striatal activity integrates punishment and reward value in response to stimuli predicting mixed outcomes [45].

While the fMRI studies cited above support the notion that a single decision variable capturing both probability and magnitude is encoded in these areas, some studies have shown spatial decomposition of probability and magnitude information within the striatum $[27,32,41]$. In one example, Yacubian and colleagues asked subjects to place a bet of one of two magnitudes (either $€ 1.00$ or $€ 5.00$ ) on gambles involving a winning probability of either $12.5 \%$ or $50 \%$ [41]. Activity was analyzed during an anticipatory period, before the outcome was revealed. Probability-related activations arose in more anterior and lateral regions of the 
ventral striatum, whereas magnitude-related activations arose in more posterior and medial regions (Fig. 2). Despite this segregation, it should be kept in mind that magnitude- and probability-related activations overlap substantially in large areas of the striatum, as would be expected from a value-coding region.

\section{--- Figure 2 ---}

Moreover, fMRI activations related to the magnitude or probability of expected outcomes have also been reported in cortical regions, including anterior cingulate cortex (ACC) [14,31], posterior parietal cortex (PPC) [3,45], superior frontal [31], lateral prefrontal (LPFC) [27,4648], as well as medial (MPFC) and ventromedial prefrontal cortex (VMPFC) $[37,38,42,46,48$ 50]. The responses in orbitofrontal cortex (OFC) to rewards as well as to reward-predictive stimuli represent high versus low reward magnitude information in distributed patterns [51,51]. Thus, even though electrophysiological recordings in animals report heterogeneous signals in prefrontal regions, such that the firing rate of different value-coding neurons increases and decreases with magnitude [52], neuroimaging methods can nevertheless be used to study the representation of magnitude in such regions. Moreover, human OFC responses represent the value of rewards and punishments on a common monotonic scale [3], which enables computations across the entire value range. Common or combined coding of both components has been observed in ACC for error magnitude and probability [53], in medial OFC [31,54] and in LPFC for reward magnitude and probability [27]. By contrast, preferential activations to probability, rather than magnitude, arise in parts of the MPFC [37]. 
Different brain regions have been suggested to code value in the context of model-based (goal-directed) and model-free (habitual) behavior [55,56]. Specifically, a dissociation of these different value signals has been shown within the striatum, with activity in the caudate nucleus coding for model-based, and in the putamen for model-free value signals [56]. However, both regions are functionally connected to the VMPFC, where they can be integrated to support value-based choice. This is in line with a large number of human neuroimaging studies reporting value-related activity in the VMPFC during value-based choice [57-66].

Moreover, recent evidence suggests that different regions in the ventral PFC are involved in coding the general vs. specific value of predicted outcomes. General value signals are independent of the specific nature of the reward (e.g., cherries or elderberries) and can be used to compare and choose between alternative outcomes, but are unable to inform expectations about the specific identity of the outcome. In contrast, identity-specific representations conjointly represent information about both value (how good is it?) and outcome identity (what is it?). Human imaging work has linked activity in the VMPFC to general value signals, which can be used to compare different expected outcomes on a common scale $[59,67-70]$. In contrast, activity in the OFC has been shown to code for specific rewards [71,72], and neural value codes for different reward categories differ in this region [69].

In line with the fact that the medial and lateral OFC belong to largely separate networks $[73,74]$, the central and lateral OFC represent the value of future rewards in the form of identity-specific value codes, whereas value representations in medial areas generalize across different outcome-identities $[68,75,76]$. Such a dual representation of general and specific 
values in the PFC may allow for different forms of reward-related behaviors. Specifically, individuals may use general values to compare different options and to exert simple behaviors such as approach and avoidance responses. On the other hand, identity-specific values may support choices in line with the current needs of the organism, allow for outcome-specific responding, and enable the organism to flexibly update value predictions according to changes in the environment.

\section{SALIENCE}

\subsection{DEFINITION AND BEHAVIORAL FUNCTION}

At the most general level, salience can be defined as the capacity of a stimulus to direct attention. However, salience is an ill-defined concept in cognitive neuroscience. Part of the confusion lies in that different researchers have used the same term to refer to different ideas of what makes a stimulus salient. For instance, in visual neuroscience, the term is used to describe the perceptual salience (often saliency) of a stimulus, that is, its physical properties (i.e., the color, contrast, orientation, or luminance) that make it more likely to capture attention $[77,78]$. On the other hand, the field of associative learning and value-based decision making uses the term to describe the acquired salience of a stimulus, that is, the importance that a stimulus has acquired through association with an incentive outcome. Perceptual salience is not a topic of this chapter and instead, we will only focus on acquired salience, and define it as a function of the value of the stimulus.

More specifically, in line with associative learning theories, we define salience as the absolute (i.e., unsigned) value predicted by a stimulus (Fig. 1a). In other words, both rewarding and punishing outcomes are salient, and the degree of salience is determined by the value of the 
predicted outcome [2,3]. Without loss aversion [16], a stimulus predicting the receipt of one cherry has the same salience as a stimulus predicting the loss of one cherry. As such, salience relates to the importance of a stimulus for motivated behavior independent of its valence. The key behavioral function is therefore to guide attention to stimuli that predict motivationally relevant outcomes in order to facilitate neural processes related to planning and executing appropriate responses.

The definition of salience is straightforward for deterministic outcomes, where outcomes are predicted with no uncertainty. In the case of probabilistic stimuli, however, the definition of salience is more complicated. Specifically, probability affects salience not only by changing value (see above). In fact, the attention-capturing properties of a stimulus have historically been described as associability, a concept that is closely related to salience and is meant to describe how well associations can be formed with a stimulus. A key determinant of associability is the predictability of a stimulus, that is, the probability with which a stimulus predicts an outcome. Different mechanisms have been suggested to describe the relationship between predictability, uncertainty, and associability [10,12]. Specifically, the Mackintosh model [10] states that reliable predictors of outcomes (close to 100\%) will acquire higher salience and consequently capture more attention than poor predictors (close to $0 \%$ ), which have a low level of salience and will consequently be ignored (Fig. 1B). In contrast, the Pearce-Hall model [12] posits that a high level of salience (and thus attention) should be applied to unreliable predictors of outcomes such that learning of these is enhanced. In other words, unreliable predictors (50\%) are assumed to have higher salience than reliable predictors (close to $0 \%$ and $100 \%$, Fig. 1B). 
The models of Mackintosh, and Pearce and Hall have recently been combined by a third model of attention in associative learning [13]. The Esber-Haselgrove model [13] assumes that (1) stimuli acquire salience to the degree that they predict incentive outcomes (similar to Mackintosh), (2) that both the occurrence and non-occurrence of an incentive outcome are salient events that are both partially predicted by a non-deterministic stimulus, and (3) that salience is defined as the sum of the absolute values of all possible outcomes (occurrence and non-occurrence of positive and negative outcomes) associated with a stimulus. Based on these assumptions, this model predicts that both high (100\%) and low probability cues $(0 \%)$ have a lower salience than intermediate predictors of outcomes (e.g., 50\%), and that good predictors $(100 \%)$ have a generally higher salience than poor predictors $(0 \%)$ [79]. In other words, the Esber-Haselgrove model predicts a skewed, inverted u-shaped relationship between probability and salience (Fig. 1B).

Of note, defining salience as the sum of all (positive and negative) outcomes predicted by a stimulus is a fundamental idea, which can also be applied to deterministic predictors of outcomes. Specifically, in the case of compound stimuli, predicting multiple deterministic (or probabilistic) outcomes, salience can be defined as the absolute value of the average predicted outcome (global salience): a stimulus predicting both the receipt of one cherry and the loss of one cherry would have a global salience of zero. Alternatively, salience can be defined as the sum of the absolute values of the individual predicted outcomes (elemental salience, Fig. 3C): a stimulus predicting both the receipt of one cherry and the loss of one cherry would have an elemental salience corresponding to two cherries. Supporting the fundamental assumption of the Esber-Haselgrove model, we recently found [45] that the positive and negative outcomes predicted by individual stimuli contribute independently to the salience of the compound 
stimulus (Fig. 3D). Specifically, elemental salience predicted the latency of value-based responses and did so significantly better than global salience.

\section{--- Figure 3 ---}

\subsection{CONFOUNDS IN OPERATIONAL DEFINITIONS OF SALIENCE}

In order to test for behavioral and neural effects of salience, operational definitions of salience are required. From the paragraphs above, it should have become clear that the definition of salience is not as straightforward as intuitively assumed. Moreover, in many cases, salience is confounded by value, magnitude, or probability [80]. Specifically, in most studies using only rewards or only punishments as outcomes, value and salience are perfectly correlated (Fig. 1). Accordingly, neural signals that increase with reward magnitude could be related to value or salience $[3,81]$. This is especially true if neural responses to value and salience are heterogeneous, i.e., if the activity of different neuronal populations increases and decreases with increasing value [52]. Thus, if value is defined by different levels of outcome magnitude, experimental designs need to include both appetitive and aversive outcomes in order to dissociate value and salience.

Similarly, when varying salience as a function of value operationalized by increases in probability, salience is likely to be confounded by probability and/or risk. As can be seen in Figure 1B, in order to dissociate salience as defined in the Esber-Haselgrove model (see Figure 1 and section 3.1 for a description of the model) from coding of value and risk (i.e., salience as defined in the Pearce-Hall model) experimental designs need to vary probability in multiple steps from $0 \%$ to $100 \%$. The critical comparison is the one between $100 \%$ and $0 \%$ 
cues, which have the same risk, but different levels of salience [79]. At the same time, however, in order to control for the different value of these cues, intermediate levels of probability, which have lower value but higher salience, need to be tested. Alternatively, in order to dissociate salience as defined in the Mackintosh model from value and risk, experimental designs need to cover multiple steps of probability from $0 \%$ to $100 \%$ and must include both positive and negative outcomes. Here, value increases and decreases with the probability of positive and negative outcomes, respectively, whereas salience increases with the probability of both appetitive and aversive outcomes.

Importantly, while it is theoretically possible to define salience in terms of absolute value based on magnitude, probability or both (expected value), it is not clear whether these different operationalizations are computationally equivalent. Also, while both have been shown to correlate with response times, and thus appear to facilitate behavior and neural processing $[3,45,79,82]$, it is unclear whether they are processed in the same brain regions, and whether they subserve the same behavioral functions.

\subsection{SALIENCE SIGNALS IN THE HUMAN BRAIN}

Given the numerous potential confounds of salience described above, experimental investigations of salience can be challenging. Accordingly, only relatively few imaging studies have comprehensively addressed how value vs. salience are coded in the human brain. For instance, one study [82] used a simple value-based choice task involving images of snack items to dissociate value from salience. Specifically, subjects were asked to make a binding decision about whether or not they wanted to eat the currently displayed food item by choosing "Strong No", "No", "Yes", or "Strong Yes". Thus, by choosing "Yes", they could 
obtain rewards, whereas by choosing "No" they could avoid punishments. The choices were used to determine the value of the items by assigning $-2,-1,+1$ and +2 to the four answers, respectively. In line with the above magnitude-based definition, salience was defined as absolute value. The study identified several brain regions in which fMRI activity was correlated with salience, including the ACC extending to supplemental motor area, the precentral gyrus, the insula, and the fusiform gyrus. In contrast, correlations with value were found in the VMPFC extending into MPFC, in the precuneus, and in the ventral striatum.

A general problem of experiments involving choices is that aversive outcomes can be avoided. Specifically, the possibility of avoiding potential harm is likely to change the value associated with them [50]. Accordingly, it is unclear whether value and salience signals identified in studies involving choices reflect the same processes as in Pavlovian tasks, where outcomes cannot be avoided. Moreover, decisions about stimuli with low absolute values are intrinsically more difficult because the values are closer to indifference than choices involving high absolute values, and salience signals in choice paradigms are therefore correlated with difficulty and nonspecifically enhanced processing. Finally, anticipatory outcome signals related to value and salience are related to neural processing of action selection and choice execution, preventing a straightforward interpretation of these signals.

In order to clearly dissociate neural representations of value and salience, we recently used a non-instrumental task, involving low and high magnitude monetary rewards and punishments [3]. Besides dissociating value and salience, this task controlled for several additional confounds. First, because of the non-instrumental nature of the task, aversive outcomes could not be avoided, thus maintaining the salience and value of the aversive stimuli, and at the same time preventing choice-related confounds. Second, we used abstract visual stimuli and 
associated them with rewarding ( +0.5 or +5 Euros) and punishing $(-0.5$ or -5 Euros) outcomes using classical conditioning (Fig. 4A). This controlled for preexisting associations of the stimuli, and thus ensured approximately equal value of the outcomes across subjects. Furthermore, instead of using univariate fMRI data analysis, we used multivoxel pattern analysis (MVPA) techniques $[83,84]$, which are sensitive to distributed representations.

Our results revealed that fMRI activity patterns in the OFC, which had been assumed to be related to value $[51,69]$ indeed represent value, and not salience (Fig. 4C). Moreover, also activity patterns in the intraparietal sulcus (IPS) were related to value (Fig. 4D), which is noteworthy because previously reported value signals in the IPS [85-87] had been reinterpreted as salience signals [81]. Thus, in the OFC and the posterior parietal cortex, distributed signals decreased with the magnitude of predicted punishments and increased with the magnitude of predicted rewards, reflecting a value signal. In contrast, we found salience signals in the ACC (Fig. 4E) and the inferior parietal cortex, extending into the temporoparietal junction (TPJ; Fig. 4D). Salience signals also emerged in more superior regions of the parietal cortex, overlapping with neural representations of value in the IPS (Fig. 4D). These results demonstrate that value and salience can be processed in the very same brain region, highlighting the importance of experimentally dissociating both variables.

\section{--- Figure 4 ---}

Taken together, the results from this study using deterministic outcome magnitude to vary salience identified a network including the ACC and the TPJ that is involved in salience processing. Interestingly, the TPJ has been suggested to be part of a ventral attention network 
that is involved in mediating orienting responses toward behaviorally relevant stimuli $[88,89]$. Paralleling results from studies on acquired salience discussed above, experiments found that the TPJ responds to behaviorally relevant stimuli [90], even if these stimuli are of low visual salience or unattended [91].

Our earlier study [45] converged with the subsequent one by showing salience signals in the TPJ. Specifically, similar to response times, fMRI signals in the TPJ were best explained by the summed salience of all positive and negative outcomes associated with the individual cues in the compound (Fig. 3E). Moreover, functional connectivity analyses suggested that salience signals in the TPJ were functionally linked to activity in the locus coeruleus (LC, Fig. 3G), a noradrenergic nucleus in the brainstem [45]. This finding is in line with the hypothesis that the TPJ's role in redirecting attention is supported by the activity of noradrenergic neurons in the LC [89], which directly innervate the superior temporal gyrus and inferior parietal cortex $[92,93]$. Noradrenaline has long been implicated in arousal and attentionrelated increases in behavioral performance. In particular, noradrenergic neurons in the LC respond to target cues with phasic activity increases [94], and the magnitude of this response is correlated with measures of task performance [95]. These findings contrast with striatal value signals that are presumably related to midbrain dopamine (Fig. 3F).

A few studies have investigated brain signals that correlate with salience, defined as a function of probability rather than magnitude. For instance, using an associative learning task with positive and negative outcomes (appetitive juice, money, salt water, and aversive pictures), one study showed that during learning activity in the VMPFC was correlated with the salience of stimuli that predicted positive and negative outcomes with $50 \%$ contingency [96]. Because activity for stimuli predicting positive and negative outcomes had the same 
sign, and thus cannot be explained by value (Fig. 1B), this finding suggests that VMPFC activity correlates with the acquired salience of predicted outcomes (Fig. 5). However, even though this design controlled well for value, it only included stimuli that predicted the outcome with $50 \%$ contingency, and thus, it is unclear whether the observed VMPFC signals reflect salience or risk. Nevertheless, a similar finding of salience in the VMPFC has been reported in a study that operationalized salience as $80 \%$ vs. $20 \%$ probability of reward or punishment [97]. Thus, levels of salience were independent of risk. Moreover, the same study reported a correlation between value (defined as the product of probability and valence and thus independent of salience) and fMRI activity in the central OFC, close to where value signals (defined as magnitude) have recently been reported [3].

\section{--- Figure 5 ---}

Taken together, these studies suggest a role for the VMPFC in salience processing, operationalized using probability. Supporting this idea, the firing patterns of neurons in the animal OFC, a region closely related to the human VMPFC, have shown to be explained by salience as defined by the Esber-Haselgrove model [79]. By contrast, studies using the magnitude of positive and negative outcomes reliably identified the ACC [82] and the TPJ $[3,45]$, whereas VMPFC activity has not been linked to salience defined in this way [82]. Given these contrasting findings, it appears that salience operationalized as a function of probability and magnitude are differently processed in the human brain (Fig. 5C). In theory, probability- and magnitude-based salience could play differential roles for learning and behavior, respectively. 


\section{CONCLUSIONS}

Value is a well understood concept that increases with magnitude and probability of reward and decreases with magnitude and probability of punishment, whereas salience has been less well defined. We show that by defining salience as absolute value, salience can be given a precise and quantifiable interpretation, both with respect to magnitude and probability. Defined in this way, salience increases with magnitude and probability of both reward and punishment. The close relation of value and salience implies that they can be separated experimentally only if both rewards and punishments are used. Human neuroimaging studies which followed this approach revealed value signals in the striatum, OFC and VMPFC, and PPC. Typically, magnitude- and probability-based value signals co-localize. By contrast, magnitude-based salience signals arose in TPJ and ACC, whereas probability-based salience signals were found in the VMPFC, suggesting different types of neural salience signals.

\section{REFERENCE LIST}

[1] E. Mayr, Behavior programs and evolutionary strategies, American Scientist. 62 (1974) 650-659.

[2] E.S. Bromberg-Martin, M. Matsumoto \& O. Hikosaka, Dopamine in motivational control: rewarding, aversive, and alerting, Neuron. 68 (2010) 815-834.

[3] T. Kahnt, S.Q. Park, J.D. Haynes \& P.N. Tobler, Disentangling neural representations of value and salience in the human brain, Proc Natl Acad Sci U S A. 111 (2014) 50005005 .

[4] J.P. O'Doherty, The problem with value, Neurosci Biobehav Rev. 43 (2014) 259-268.

[5] W. Schultz, Updating dopamine reward signals, Curr Opin Neurobiol. 23 (2013) 229238.

[6] A. Dickinson, Contemporary Animal Learning Theory (Problems in the Behavioural Sciences), Cambridge University Press, New York, 1981.

[7] P. Pavlov, Conditioned reflexes: An investigation of the physiological activity of the cerebral cortex (G. V. Anrep, Trans.), Oxford Univ. Press, London, 1927.

[8] E. Thorndike, Animal Intelligence: Experimental Studies, MacMillan, New York, 1911. 
[9] R.A. Rescorla, A.R. Wagner, A theory of Pavlovian conditioning: variations in the effectiveness of reinforcement and nonreinforcement., in: Black AH, Prokasy WF (Eds.), Classical Conditioning II: Current Research and Theory, Appleton Century Crofts, New York, 1972.

[10] N.J. Mackintosh, Theory of Attention - Variations in Associability of Stimuli with Reinforcement, Psychol Rev. 82 (1975) 276-298.

[11] C.J. Mitchell, M.E. Le Pelley, Attention and associative learning: from brain to behaviour, Oxford University Press, Oxford, UK, 2010.

[12] J.M. Pearce \& G. Hall, A model for Pavlovian learning: variations in the effectiveness of conditioned but not of unconditioned stimuli, Psychol Rev. 87 (1980) 532-552.

[13] G.R. Esber \& M. Haselgrove, Reconciling the influence of predictiveness and uncertainty on stimulus salience: a model of attention in associative learning, Proc Biol Sci. 278 (2011) 2553-2561.

[14] J. Fujiwara, P.N. Tobler, M. Taira, T. Iijima \& K. Tsutsui, Segregated and integrated coding of reward and punishment in the cingulate cortex, J Neurophysiol. 101 (2009) 3284-3293.

[15] P.N. Tobler, A. Dickinson \& W. Schultz, Coding of predicted reward omission by dopamine neurons in a conditioned inhibition paradigm, J Neurosci. 23 (2003) 10402 10410.

[16] D. Kahneman \& A. Tversky, Prospect Theory - Analysis of Decision Under Risk, Econometrica. 47 (1979) 263-291.

[17] M. Cabanac, Pleasure: the common currency, J Theor Biol. 155 (1992) 173-200.

[18] G. Bower \& M. Tarpold, Reward magnitude and learning in a single-presentation discrimination, J Comp Physiol Psychol. 52 (1959) 727-729.

[19] P.N. Tobler, E.U. Weber, Valuation of risky and uncertain choices, in: P.W. Glimcher, E. Fehr (Eds.), Neuroeconomics, Academic Press, Oxford, 2013. 149-172.

[20] J. von Neumann, O. Morgenstern, Theory of Games and Economic Behavior, 2 ed. Princeton University Press, Princeton, 1947.

[21] N.D. Daw, P.N. Tobler, Value learning through reinforcement: The basics of dopamine and reinforcement learning, in: P.W. Glimcher, E. Fehr (Eds.), Neuroeconomics, Academic Press, Oxford, 2013. 283-298.

[22] R. Sutton, A. Barto, Reinforcement Learning: An Introduction, MIT Press, Cambridge, MA, 1998.

[23] R.J. Dolan \& P. Dayan, Goals and habits in the brain, Neuron. 80 (2013) 312-325.

[24] E. Duzel, N. Bunzeck, M. Guitart-Masip, B. Wittmann, B.H. Schott \& P.N. Tobler, Functional imaging of the human dopaminergic midbrain, Trends Neurosci. 32 (2009) 321-328. 
[25] P.N. Tobler, C.D. Fiorillo \& W. Schultz, Adaptive coding of reward value by dopamine neurons, Science. 307 (2005) 1642-1645.

[26] C.D. Fiorillo, P.N. Tobler \& W. Schultz, Discrete coding of reward probability and uncertainty by dopamine neurons, Science. 299 (2003) 1898-1902.

[27] P.N. Tobler, J.P. O'Doherty, R.J. Dolan \& W. Schultz, Reward value coding distinct from risk attitude-related uncertainty coding in human reward systems, $\mathrm{J}$ Neurophysiol. 97 (2007) 1621-1632.

[28] B. Abler, H. Walter, S. Erk, H. Kammerer \& M. Spitzer, Prediction error as a linear function of reward probability is coded in human nucleus accumbens, Neuroimage. 31 (2006) 790-795.

[29] A. Shenhav \& J.D. Greene, Moral judgments recruit domain-general valuation mechanisms to integrate representations of probability and magnitude, Neuron. 67 (2010) 667-677.

[30] P.N. Tobler, G.I. Christopoulos, J.P. O'Doherty, R.J. Dolan \& W. Schultz, Neuronal distortions of reward probability without choice, J Neurosci. 28 (2008) 11703-11711.

[31] B. Studer, A.M. pergis-Schoute, T.W. Robbins \& L. Clark, What are the Odds? The Neural Correlates of Active Choice during Gambling, Front Neurosci. 6 (2012) 46.

[32] G.S. Berns \& E. Bell, Striatal topography of probability and magnitude information for decisions under uncertainty, Neuroimage. 59 (2012) 3166-3172.

[33] M. Hsu, M. Bhatt, R. Adolphs, D. Tranel \& C.F. Camerer, Neural systems responding to degrees of uncertainty in human decision-making, Science. 310 (2005) 1680-1683.

[34] I. Levy, J. Snell, A.J. Nelson, A. Rustichini \& P.W. Glimcher, Neural representation of subjective value under risk and ambiguity, J Neurophysiol. 103 (2010) 1036-1047.

[35] C.J. Burke \& P.N. Tobler, Reward skewness coding in the insula independent of probability and loss, J Neurophysiol. 106 (2011) 2415-2422.

[36] B. Knutson, C.M. Adams, G.W. Fong \& D. Hommer, Anticipation of increasing monetary reward selectively recruits nucleus accumbens, J Neurosci. 21 (2001) RC159.

[37] B. Knutson, J. Taylor, M. Kaufman, R. Peterson \& G. Glover, Distributed neural representation of expected value, J Neurosci. 25 (2005) 4806-4812.

[38] S.M. Tom, C.R. Fox, C. Trepel \& R.A. Poldrack, The neural basis of loss aversion in decision-making under risk, Science. 315 (2007) 515-518.

[39] K. Preuschoff, P. Bossaerts \& S.R. Quartz, Neural differentiation of expected reward and risk in human subcortical structures, Neuron. 51 (2006) 381-390.

[40] J. Yacubian, J. Glascher, K. Schroeder, T. Sommer, D.F. Braus \& C. Buchel, Dissociable systems for gain- and loss-related value predictions and errors of prediction in the human brain, J Neurosci. 26 (2006) 9530-9537. 
[41] J. Yacubian, T. Sommer, K. Schroeder, J. Glascher, D.F. Braus \& C. Buchel, Subregions of the ventral striatum show preferential coding of reward magnitude and probability, Neuroimage. 38 (2007) 557-563.

[42] H.C. Breiter, I. Aharon, D. Kahneman, A. Dale \& P. Shizgal, Functional imaging of neural responses to expectancy and experience of monetary gains and losses, Neuron. 30 (2001) 619-639.

[43] G.I. Christopoulos, P.N. Tobler, P. Bossaerts, R.J. Dolan \& W. Schultz, Neural correlates of value, risk, and risk aversion contributing to decision making under risk, J Neurosci. 29 (2009) 12574-12583.

[44] M.R. Delgado, V.A. Stenger \& J.A. Fiez, Motivation-dependent responses in the human caudate nucleus, Cereb Cortex. 14 (2004) 1022-1030.

[45] T. Kahnt \& P.N. Tobler, Salience signals in the right temporoparietal junction facilitate value-based decisions, J Neurosci. 33 (2013) 863-869.

[46] H. Plassmann, J. O'Doherty \& A. Rangel, Orbitofrontal cortex encodes willingness to pay in everyday economic transactions, J Neurosci. 27 (2007) 9984-9988.

[47] P.N. Tobler, G.I. Christopoulos, J.P. O'Doherty, R.J. Dolan \& W. Schultz, Riskdependent reward value signal in human prefrontal cortex, Proc Natl Acad Sci U S A. 106 (2009) 7185-7190.

[48] H. Plassmann, J.P. O'Doherty \& A. Rangel, Appetitive and aversive goal values are encoded in the medial orbitofrontal cortex at the time of decision making, J Neurosci. 30 (2010) 10799-10808.

[49] T. Kahnt, J. Heinzle, S.Q. Park \& J.D. Haynes, The neural code of reward anticipation in human orbitofrontal cortex, Proc Natl Acad Sci U S A. 107 (2010) 6010-6015.

[50] H. Kim, S. Shimojo \& J.P. O'Doherty, Is Avoiding an Aversive Outcome Rewarding? Neural Substrates of Avoidance Learning in the Human Brain, PLoS Biol. 4 (2006) e233.

[51] T. Kahnt, J. Heinzle, S.Q. Park \& J.D. Haynes, Decoding the Formation of Reward Predictions across Learning, J Neurosci. 31 (2011) 14624-14630.

[52] S.W. Kennerley, A.F. Dahmubed, A.H. Lara \& J.D. Wallis, Neurons in the Frontal Lobe Encode the Value of Multiple Decision Variables, J Cogn Neurosci. 21 (2009) 1162-1178.

[53] J.W. Brown \& T.S. Braver, Risk prediction and aversion by anterior cingulate cortex, Cogn Affect Behav Neurosci. 7 (2007) 266-277.

[54] M. Symmonds, P. Bossaerts \& R.J. Dolan, A behavioral and neural evaluation of prospective decision-making under risk, J Neurosci. 30 (2010) 14380-14389.

[55] D. McNamee, M. Liljeholm, O. Zika \& J.P. O'Doherty, Characterizing the associative content of brain structures involved in habitual and goal-directed actions in humans: a multivariate FMRI study, J Neurosci. 35 (2015) 3764-3771. 
[56] K. Wunderlich, P. Dayan \& R.J. Dolan, Mapping value based planning and extensively trained choice in the human brain, Nat Neurosci. 15 (2012) 786-791.

[57] T.A. Hare, C.F. Camerer \& A. Rangel, Self-control in decision-making involves modulation of the vmPFC valuation system, Science. 324 (2009) 646-648.

[58] J.W. Kable \& P.W. Glimcher, The neurobiology of decision: consensus and controversy, Neuron. 63 (2009) 733-745.

[59] D.J. Levy \& P.W. Glimcher, The root of all value: a neural common currency for choice, Curr Opin Neurobiol. 22 (2012) 1027-1038.

[60] K. Wunderlich, A. Rangel \& J.P. O'Doherty, Neural computations underlying actionbased decision making in the human brain, Proc Natl Acad Sci U S A. 106 (2009) 17199-17204.

[61] H.C. Barron, R.J. Dolan \& T.E. Behrens, Online evaluation of novel choices by simultaneous representation of multiple memories, Nat Neurosci. 16 (2013) 14921498

[62] T.E. Behrens, M.W. Woolrich, M.E. Walton \& M.F. Rushworth, Learning the value of information in an uncertain world, Nat Neurosci. 10 (2007) 1214-1221.

[63] M.F. Rushworth, M.P. Noonan, E.D. Boorman, M.E. Walton \& T.E. Behrens, Frontal cortex and reward-guided learning and decision-making, Neuron. 70 (2011) 10541069.

[64] J.A. Clithero \& A. Rangel, Informatic parcellation of the network involved in the computation of subjective value, Soc Cogn Affect Neurosci (2013)

[65] O. Bartra, J.T. McGuire \& J.W. Kable, The valuation system: a coordinate-based meta-analysis of BOLD fMRI experiments examining neural correlates of subjective value, Neuroimage. 76 (2013) 412-427.

[66] S.Q. Park, T. Kahnt, J. Rieskamp \& H.R. Heekeren, Neurobiology of value integration: when value impacts valuation, J Neurosci. 31 (2011) 9307-9314.

[67] J. Chikazoe, D.H. Lee, N. Kriegeskorte \& A.K. Anderson, Population coding of affect across stimuli, modalities and individuals, Nat Neurosci. 17 (2014) 1114-1122.

[68] J.D. Howard, J.A. Gottfried, P.N. Tobler \& T. Kahnt, Identity-specific coding of future rewards in the human orbitofrontal cortex, Proc Natl Acad Sci U S A. 112 (2015) 5195-5200.

[69] D. McNamee, A. Rangel \& J.P. O'Doherty, Category-dependent and categoryindependent goal-value codes in human ventromedial prefrontal cortex, Nat Neurosci. 16 (2013) 479-485.

[70] V.S. Chib, A. Rangel, S. Shimojo \& J.P. O'Doherty, Evidence for a common representation of decision values for dissimilar goods in human ventromedial prefrontal cortex, J Neurosci. 29 (2009) 12315-12320. 
[71] M.C. Klein-Flugge, H.C. Barron, K.H. Brodersen, R.J. Dolan \& T.E. Behrens, Segregated Encoding of Reward-Identity and Stimulus-Reward Associations in Human Orbitofrontal Cortex, J Neurosci. 33 (2013) 3202-3211.

[72] G. Sescousse, J. Redoute \& J.C. Dreher, The architecture of reward value coding in the human orbitofrontal cortex, J Neurosci. 30 (2010) 13095-13104.

[73] T. Kahnt, L.J. Chang, S.Q. Park, J. Heinzle \& J.D. Haynes, Connectivity-Based Parcellation of the Human Orbitofrontal Cortex, J Neurosci. 32 (2012) 6240-6250.

[74] D. Ongur \& J.L. Price, The organization of networks within the orbital and medial prefrontal cortex of rats, monkeys and humans, Cereb Cortex. 10 (2000) 206-219.

[75] Y. Li, G. Sescousse, C. Amiez \& J.C. Dreher, Local morphology predicts functional organization of experienced value signals in the human orbitofrontal cortex, $\mathrm{J}$ Neurosci. 35 (2015) 1648-1658.

[76] G. Sescousse, X. Caldu, B. Segura \& J.C. Dreher, Processing of primary and secondary rewards: a quantitative meta-analysis and review of human functional neuroimaging studies, Neurosci Biobehav Rev. 37 (2013) 681-696.

[77] C. Bogler, S. Bode \& J.D. Haynes, Decoding successive computational stages of saliency processing, Curr Biol. 21 (2011) 1667-1671.

[78] L. Itti \& C. Koch, Computational modelling of visual attention, Nat Rev Neurosci. 2 (2001) 194-203.

[79] M. Ogawa, M.A. van der Meer, G.R. Esber, D.H. Cerri, T.A. Stalnaker \& G. Schoenbaum, Risk-responsive orbitofrontal neurons track acquired salience, Neuron. 77 (2013) 251-258.

[80] M.R. Roesch, D.J. Calu, G.R. Esber \& G. Schoenbaum, All that glitters ... dissociating attention and outcome expectancy from prediction errors signals, J Neurophysiol. 104 (2010) 587-595.

[81] M.L. Leathers \& C.R. Olson, In monkeys making value-based decisions, LIP neurons encode cue salience and not action value, Science. 338 (2012) 132-135.

[82] A. Litt, H. Plassmann, B. Shiv \& A. Rangel, Dissociating valuation and saliency signals during decision-making, Cereb Cortex. 21 (2011) 95-102.

[83] K.A. Norman, S.M. Polyn, G.J. Detre \& J.V. Haxby, Beyond mind-reading: multivoxel pattern analysis of fMRI data, Trends Cogn Sci. 10 (2006) 424-430.

[84] J.D. Haynes \& G. Rees, Decoding mental states from brain activity in humans, Nat Rev Neurosci. 7 (2006) 523-534.

[85] M.L. Platt \& P.W. Glimcher, Neural correlates of decision variables in parietal cortex, Nature. 400 (1999) 233-238.

[86] C.J. Peck, D.C. Jangraw, M. Suzuki, R. Efem \& J. Gottlieb, Reward modulates attention independently of action value in posterior parietal cortex, J Neurosci. 29 (2009) 11182-11191. 
[87] K. Louie \& P.W. Glimcher, Separating value from choice: delay discounting activity in the lateral intraparietal area, J Neurosci. 30 (2010) 5498-5507.

[88] M. Corbetta \& G.L. Shulman, Control of goal-directed and stimulus-driven attention in the brain, Nat Rev Neurosci. 3 (2002) 201-215.

[89] M. Corbetta, G. Patel \& G.L. Shulman, The reorienting system of the human brain: from environment to theory of mind, Neuron. 58 (2008) 306-324.

[90] J.J. Geng \& G.R. Mangun, Right temporoparietal junction activation by a salient contextual cue facilitates target discrimination, Neuroimage. 54 (2011) 594-601.

[91] I. Indovina \& E. Macaluso, Dissociation of stimulus relevance and saliency factors during shifts of visuospatial attention, Cereb Cortex. 17 (2007) 1701-1711.

[92] S.L. Foote \& J.H. Morrison, Extrathalamic modulation of cortical function, Annu Rev Neurosci. 10 (1987) 67-95.

[93] J.H. Morrison \& S.L. Foote, Noradrenergic and serotoninergic innervation of cortical, thalamic, and tectal visual structures in Old and New World monkeys, J Comp Neurol. 243 (1986) 117-138.

[94] G. Aston-Jones, J. Rajkowski, P. Kubiak \& T. Alexinsky, Locus coeruleus neurons in monkey are selectively activated by attended cues in a vigilance task, J Neurosci. 14 (1994) 4467-4480.

[95] M. Usher, J.D. Cohen, D. Servan-Schreiber, J. Rajkowski \& G. Aston-Jones, The role of locus coeruleus in the regulation of cognitive performance, Science. 283 (1999) 549-554.

[96] E. Metereau \& J.C. Dreher, The medial orbitofrontal cortex encodes a general unsigned value signal during anticipation of both appetitive and aversive events, Cortex. 63 (2015) 42-54.

[97] M. Rothkirch, K. Schmack, F. Schlagenhauf \& P. Sterzer, Implicit motivational value and salience are processed in distinct areas of orbitofrontal cortex, Neuroimage. 62 (2012) 1717-1725.

[98] M. O'Neill \& W. Schultz, Coding of reward risk by orbitofrontal neurons is mostly distinct from coding of reward value, Neuron. 68 (2010) 789-800. 


\section{FigURE LEGENDS}
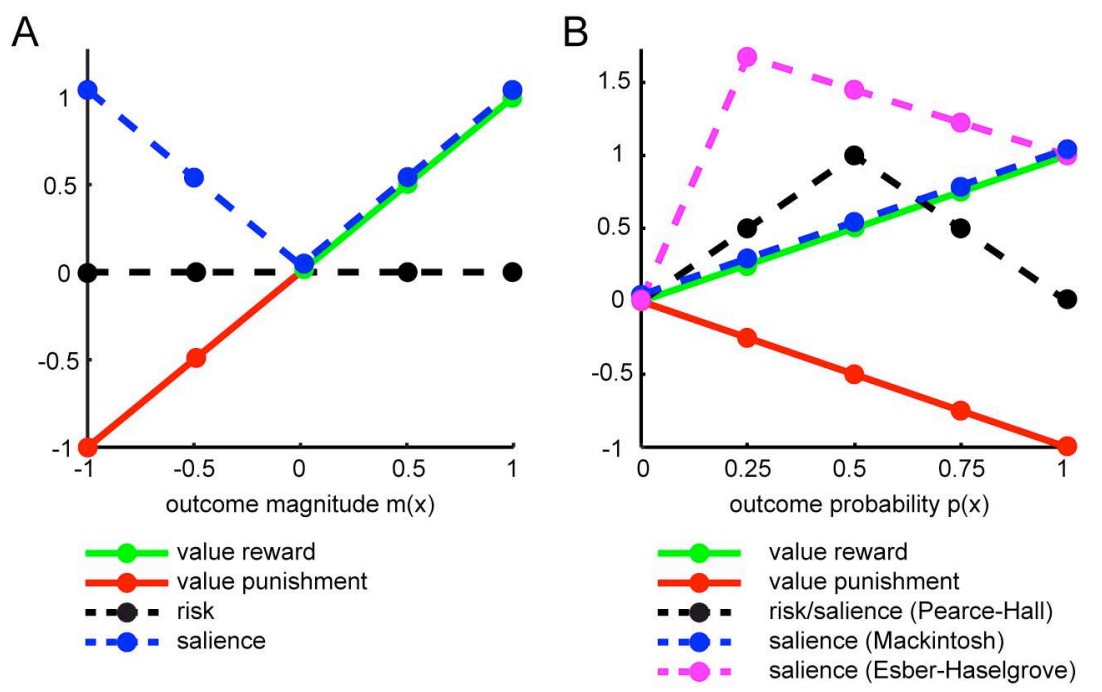

\section{FIGURE 1. OPERATIONAL DEFINITIONS OF VALUE AND SALIENCE AS A FUNCTION OF}

MAGNitUde AND PROBAbiLITY. A. Positive value, negative value, risk, and salience of deterministic predicted outcomes of different magnitudes $(-1,-0.5,0,0.5,1$ in arbitrary units).

B. Positive value, negative value, risk (salience according to the model by Pearce and Hall), salience according to the model by Mackintosh, and salience according to the model by Esber and Haselgrove of predicted outcomes (rewards and punishments) of different probabilities $(0 \%, 25 \%, 50 \%, 75 \%$, and $100 \%)$. Note, in B, Esber-Haselgrove salience is generated using the reduced model (assuming complete learning) with parameters from [79], resulting in a peak at 0.25 ; in $\mathrm{A}$ and $\mathrm{B}$, risk is defined as variance, following the tradition of finance theory $[19,98]$. 
A

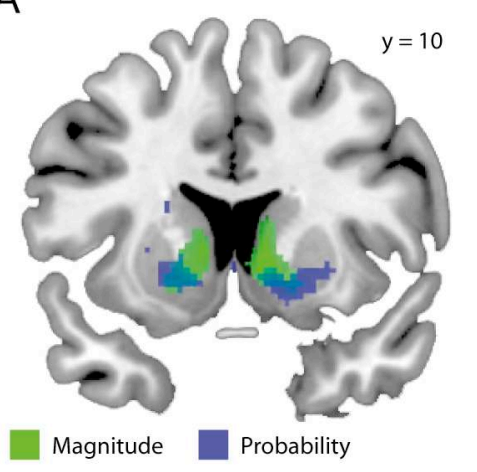

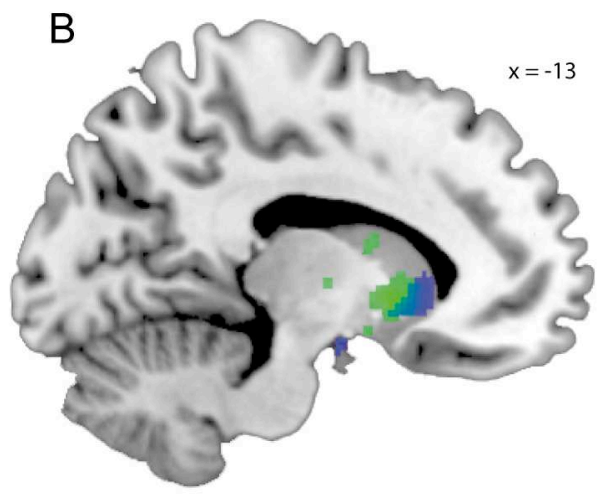

FIGURE 2. SEGREGATED ENCODING OF PROBABILITY AND MAGNITUDE IN THE VENTRAL

STRIATUM. A+B. Coronal and sagittal sections depicting magnitude and probability related fMRI activity (average of 98 subjects) in green and blue, respectively. Adapted from [41]. 

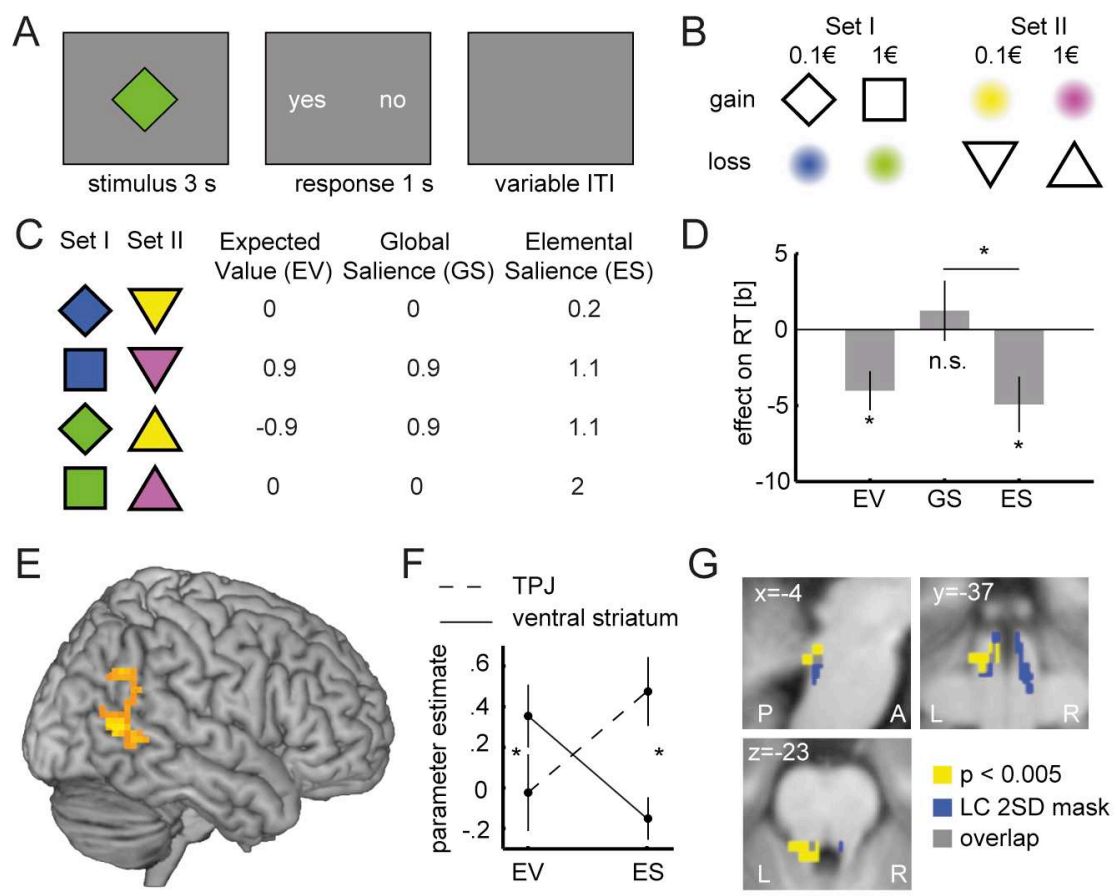

Figure 3. Salience and value of Compound Stimuli. A-C. Trial structure and experimental stimuli. Subjects underwent classical conditioning with the single stimuli depicted in $\mathbf{B}$. These stimuli were then combined into compound stimuli (depicted in $\mathbf{C}$ ) that independently varied as a function of expected value (EV, i.e. value $1+$ value 2$)$, global salience (GS, absolute value of EV, i.e. |value 1 + value 2|), and elemental salience (ES, sum of absolute values of single stimuli, i.e. $\mid$ value $1|+|$ value $2 \mid)$. D. Response times (RT) in the choice task were independently predicted by EV and ES, but did not correlate with GS. E. FMRI activity in the right temporoparietal junction (TPJ) was best correlated with elemental salience. F. Double dissociation between value-related activity in the ventral striatum and salience-related activity in the TPJ. G. Functional connectivity between the TPJ and locus coeruleus (LC) was related to elemental salience. Adapted from [45]. 

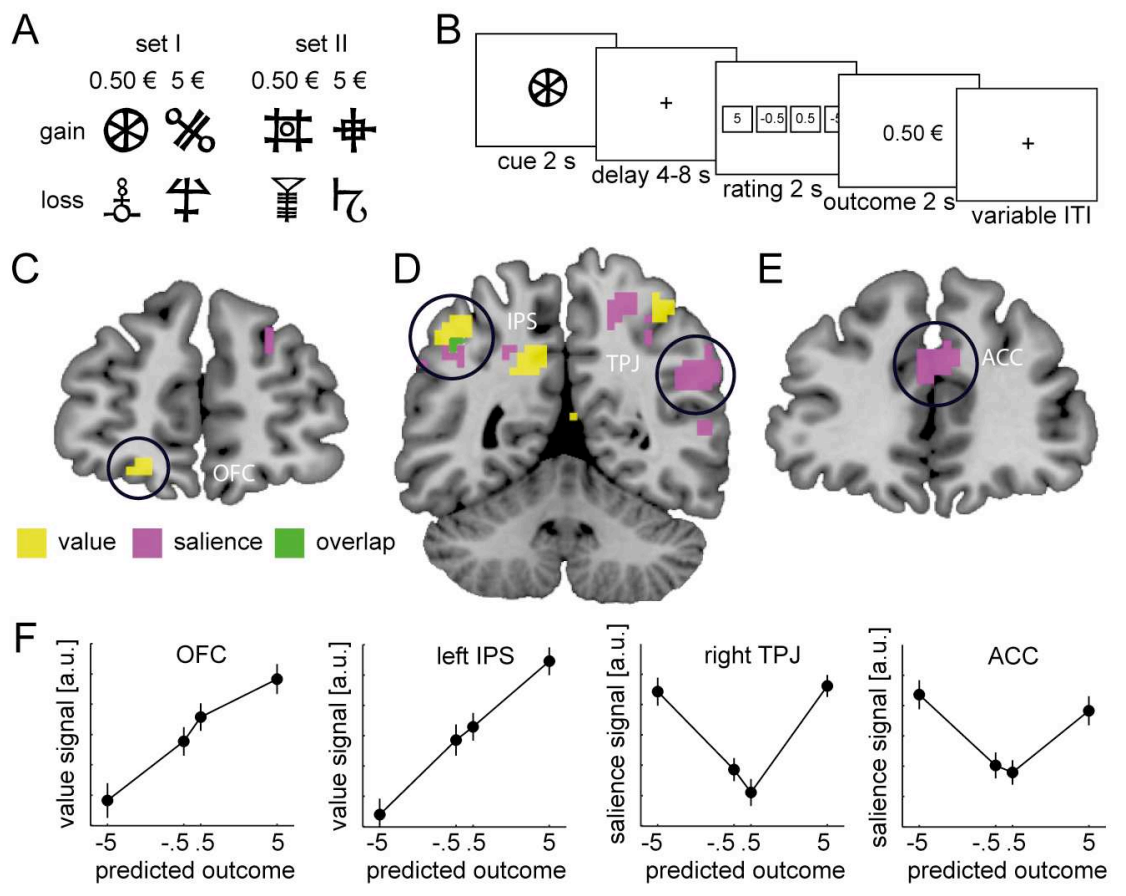

Figure 4. Brain Regions encoding Value And SAlience. A-B. Design and conditions of the non-instrumental outcome prediction task. C-E. Regions in the OFC, IPS, TPJ, and ACC, in which activity patterns correlate with the value (yellow) and salience (pink) of Pavlovian stimuli predicting rewarding and punishing outcomes in different magnitudes. F. Value and salience signals from linear support vector regression models that were trained on multivoxel activity patterns to decode value and salience of the stimuli, respectively. IPS, intraparietal sulcus; TPJ, temporoparietal junction; ACC, anterior cingulate cortex; OFC, orbitofrontal cortex. Adapted from [3]. 

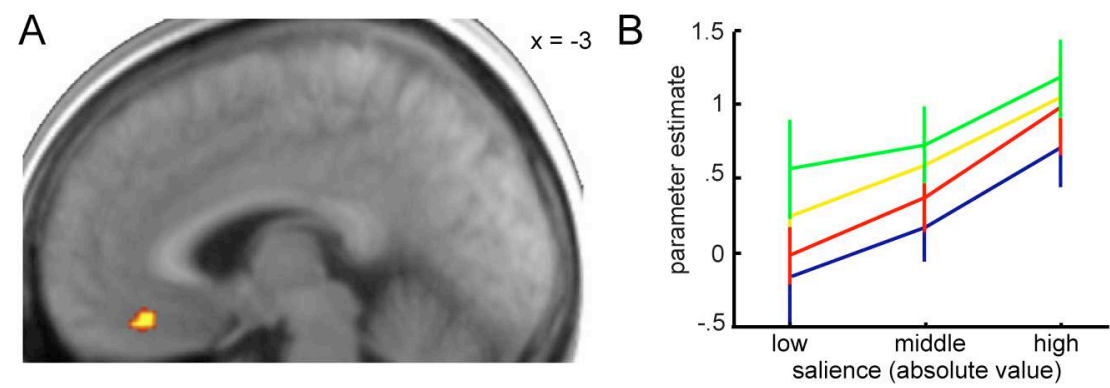

salty water apple juice

aversive picture

money
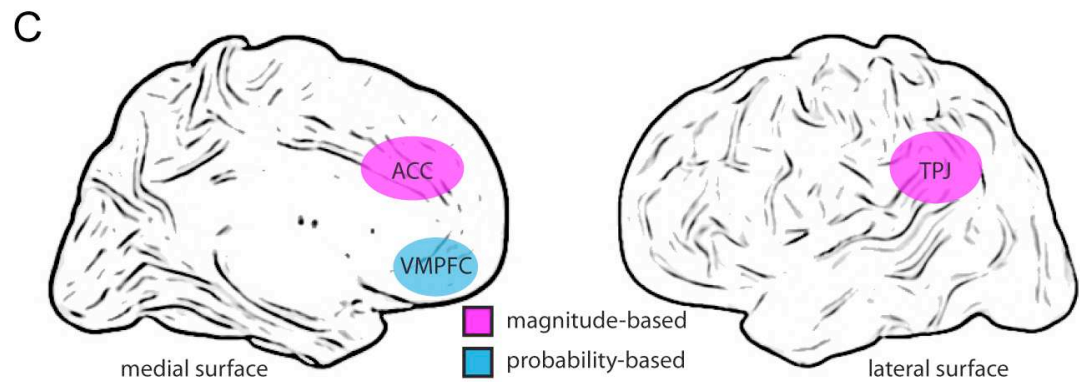

FigURE 5: Probability-based salienCe during learning. A. Activity in the ventromedial prefrontal cortex (VMPFC) correlates with the acquired probability-based salience (absolute predicted value) of different aversive and appetitive probabilistic (50\%) outcomes during associative learning. B. Parameter estimates for low, middle, and high salience of different predicted outcomes (green, salty water; yellow, apple juice; red, aversive pictures; blue, money). Adapted from [96]. C. Brain regions representing probability- and magnitude-based salience are depicted in cyan and magenta, respectively. TPJ, temporoparietal junction; ACC, anterior cingulate cortex; VMPFC, ventromedial prefrontal cortex. 